\title{
Trivium
}

Revue franco-allemande de sciences humaines et sociales - Deutsch-französische Zeitschrift für Geistesund Sozialwissenschaften

$13 \mid 2013$

Entre morale, politique et religion : la cohésion sociale selon Emile Durkheim

\section{Le dualisme de la nature humaine et ses conditions sociales et historiques}

\section{Wolfgang Schluchter}

Traducteur : Isabelle Kalinowski

\section{OpenEdition \\ Journals}

Édition électronique

URL : http://journals.openedition.org/trivium/4441

ISSN : 1963-1820

Éditeur

Les éditions de la Maison des sciences de l'Homme

Référence électronique

Wolfgang Schluchter, "Le dualisme de la nature humaine et ses conditions sociales et historiques », Trivium [En ligne], 13 | 2013, mis en ligne le 28 février 2013, consulté le 07 septembre 2020. URL : http://journals.openedition.org/trivium/4441

Ce document a été généré automatiquement le 7 septembre 2020

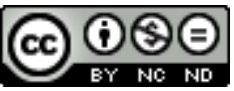

Les contenus des la revue Trivium sont mis à disposition selon les termes de la Licence Creative Commons Attribution - Pas d'Utilisation Commerciale - Pas de Modification 4.0 International. 


\title{
Le dualisme de la nature humaine et ses conditions sociales et historiques
}

\author{
Wolfgang Schluchter
}

Traduction : Isabelle Kalinowski

\section{NOTE DE L'ÉDITEUR}

Nous remercions $\mathrm{M}$. Wolfgang Schluchter ainsi que la maison d'édition Mohr-Siebeck de nous avoir accordé l'autorisation de traduire ce texte pour le présent numéro. Wir danken Herrn Wolfgang Schluchter und dem Verlag Mohr-Siebeckfür die freundliche Genehmigung, diesen Artikel in französischer Übersetzung zu publizieren.

Durkheim affirme qu'au commencement, presque tout était religion ${ }^{1}$. Le commencement correspondait pour lui à la configuration à laquelle aboutissait l'observateur sociologue en cherchant le terme le plus simple. Le plus simple, avait-il expliqué dans Les Règles de la méthode sociologique, était la plus petite unité, celle qui ne pouvait être ramenée à aucune autre sans se désagréger. A l'époque des Règles, Durkheim songeait à la horde ou au clan. Or, ce qu'il appliquait à la structure (sociale) valait aussi pour la culture, sous la figure de la religion. Le commencement de la religion correspondait donc, selon lui, à la forme la plus simple, celle qui ne pouvait être ramenée à aucune forme antérieure plus élémentaire.

2 Telle était l'approche choisie par Durkheim dans sa dernière grande monographie, consacrée en 1912 aux formes élémentaires de la vie religieuse, dont l'élaboration avait été préparée par la publication d'articles et de comptes rendus ${ }^{2}$. Entre temps, il avait élargi et approfondi sa définition du fait religieux proposée en 1899 mais il ne l'avait aucunement remise en cause. Il continuait d'appréhender les faits religieux, à l'instar de tous les faits sociaux, comme le résultat d'associations, "d'une immense coopération, qui s'étend non seulement dans l'espace, mais aussi dans le temps ${ }^{3} »$. Il ne 
cessait de les définir comme des faits sociaux dotés d'un aspect cognitif et d'un aspect normatif. En effet, tout comme les premiers systèmes d'actions, les premiers systèmes de pensée étaient pour lui d'origine religieuse : «Il n'est pas de religion qui ne soit une cosmologie en même temps qu'une spéculation sur le divin ${ }^{4} »$. Sur le divin, notons-le bien, et pas nécessairement sur Dieu ou les dieux. En effet, les religions connaissaient l'un et l'autre : la personnalisation de l'être suprême dont nous nous sentons tributaires n'était donc pas, selon Durkheim, le critère de définition décisif du fait religieux. Cela aussi, le sociologue l'avait déjà noté dans sa première tentative de définition, en se référant au bouddhisme. Par suite, le point de vue qu'il privilégiait dans la définition du fait religieux était la distinction entre le sacré et le profane. Les faits religieux, même simples, procédaient selon lui de convictions religieuses et de rites destinés à creuser un fossé infranchissable entre le sacré et le profane et, en même temps, à permettre de le franchir. Il en allait de même des faits religieux complexes, notamment dans les religions élevées : «Toutes les croyances religieuses connues, qu'elles soient simples ou complexes, présentent un même caractère commun : elles supposent une classification des choses, réelles ou idéales, que se représentent les hommes, en deux classes, en deux genres opposés, désignés généralement par deux termes distincts que traduisent assez bien les mots de profaneet de sacré5 ${ }^{\prime}$. Cependant, tout ou presque était susceptible de devenir sacré : « un rocher, un arbre, une source, un caillou, une pièce de bois, une maison, en un mot une chose quelconque peut être sacrée » - et aussi, justement, une personne ou un dieu ${ }^{6}$.

Deux genres opposés : telle est, ici, la mention la plus significative. En effet, pour Durkheim, il n'en va pas seulement, dans la distinction entre sacré et profane, des deux faces d'un seul et même phénomène, ni même d'une hiérarchie entre deux domaines de réalités qui se constitueraient par là-même, mais d'une altérité absolue. Le sacré est un domaine de réalité spécifique, qui tend à s'opposer au profane sur un mode antithétique. L'individu, au demeurant, participe de ces deux domaines de réalité. Mais ce n'est qu'à partir du moment où le sacré est organisé qu'il devient religion. Dans sa première définition du fait religieux, Durkheim n'avait pas prêté attention à ce trait caractéristique. D'où, peut-être, l'idée qu'il avait formulée dans la préface de la deuxième édition des Règles de la méthode sociologique : la sociologie est la science des institutions, de leur genèse et de leurs effets ${ }^{7}$. Quoi qu'il en soit, la définition élargie et approfondie du fait religieux tient compte de cet aspect. La voici : «Une religion est un système solidaire de croyances et de Pratiques relatives à des choses sacrées, c'est-àdire séparées, interdites, croyances et pratiques qui unissent en une même communauté morale, appelée Église, tous ceux qui y adhèrent ${ }^{8}$.»

Durkheim souligne que les aspects symboliques et institutionnels sont d'égale importance. Ce n'est qu'avec «l'Eglise » que les représentations religieuses et les règles d'action deviennent une "chose éminemment collective" ". Il se trouve ainsi amené, par ailleurs, à opérer une distinction entre magie et religion et à exclure la magie du domaine des affaires collectives ${ }^{10}$. [...]

5 On peut en dire autant d'une deuxième réflexion que Durkheim rattache à la distinction du sacré et du profane. En raison de la séparation, voire de la rivalité ou même de l'hostilité entre ces deux mondes, le monde religieux recèle toujours, du point de vue de Durkheim, une potentialité de refus du monde profane, en d'autres termes, une potentialité de refus du monde. Sur ce point aussi, Weber voit les choses très différemment. Chez lui en effet, le refus du monde d'inspiration religieuse présuppose 
l'évolution de la religion en religion de la délivrance. Durkheim ne s'intéressa que marginalement aux religions de la délivrance. Même le christianisme n'est évoqué que de manière sporadique ${ }^{11}$.

$6 \mathrm{Au}$ demeurant, la thèse affirmant l'altérité des deux domaines et l'antagonisme qui les oppose ne sert, chez Durkheim, qu'à faire ressortir les mécanismes sociaux qui opèrent malgré tout une médiation entre eux. En effet, l'individu doit pouvoir passer de l'un à l'autre sans que le sacré soit profané ou que le profane soit sacralisé. Durkheim est ainsi conduit à élaborer une théorie des rituels, qui décrit les modalités contrôlées en fonction desquelles le profane peut se rapprocher du sacré, puis, une fois que ce rapprochement a eu lieu, se retirer hors de la sphère sacrée, là encore sous contrôle. En effet, le sacré est objet de désir mais aussi source de périls, et l'on éprouve à son égard un sentiment ambigu, un mélange de crainte et d'amour. Les représentations religieuses définissent ce qu'est le sacré ; les règles d'action religieuses définissent quant à elles, sous la forme des rituels, la manière dont on doit entrer en contact avec le sacré, et communiquer avec lui. Dans son étude sur les formes élémentaires de la vie religieuse, Durkheim traite principalement des représentations totémiques et des rituels négatifs et positifs qui y sont associés. Cependant, l'étude de cette configuration religieuse initiale lui sert aussi de point de départ pour des considérations sur l'histoire de l'évolution qui restent largement prises, nous le verrons, dans la dichotomie entre les sociétés segmentaires et les sociétés de la division du travail.

7 [...] Pour Durkheim, la religion la plus élémentaire, qui ne peut plus être ramenée à aucune autre forme antérieure, est le totémisme ${ }^{12}$. Il "appartient» à la structure sociale du type segmentaire. Les règles de pensée qui lui correspondent avaient déjà été étudiées dans l'article de Durkheim et Mauss sur les classifications primitives. On retrouve ici les apports de cet article, mais ils sont désormais fondés sur un matériau considérablement élargi. Comme dans La Division du travail et dans l'étude sur le suicide, Durkheim évite le recours à la méthode dialectique. Il se conforme bien plutôt au principe de l'induction progressive ${ }^{13}$.

8 L'interprétation durkheimienne du totémisme, du reste, est contestée parmi les ethnologues. Lévi-Strauss, notamment, a évoqué l'illusion totémiste et même, plus généralement, la fin du totémisme. Il s'agissait selon lui d'une invention d'ethnologues, qui ne correspondait à rien dans la réalité. Ce jugement ne visait pas seulement Durkheim, mais il s'appliquait aussi à lui ${ }^{14}$. On peut également contester la thèse selon laquelle le totémisme est la forme de religion la plus élémentaire, même s'il est vrai que Durkheim ne lui ait attribué qu'une validité relative. Tout ceci semble indiquer que cette thèse est difficile à défendre. Cependant, dans la perspective qui nous occupe, un autre aspect est plus important. La question qui nous intéresse est la suivante : si, au commencement, la religion était presque tout, pourquoi n'en va-t-il plus de même aujourd'hui? En d'autres termes : comment Durkheim envisage-t-il les changements structurels et culturels qui trouvent leur point de départ dans la religion?

Cette question n'est pas seulement la nôtre; c'est aussi celle que pose Durkheim. S'il s'intéresse aux configurations élémentaires, c'est avant tout pour mieux comprendre les configurations complexes. L'analyse de l'élémentaire est pour lui la clé de l'analyse du complexe, à supposer il est vrai qu'on ne change pas de niveau d'émergence. Dans ce cas, le sociologue doit se plier lui aussi à la règle cartésienne selon laquelle, « dans la chaîne des vérités scientifiques, le premier anneau joue un rôle prépondérant ${ }^{15}$ ». C'est justement ce qui explique le tournant de Durkheim vers la sociologie de la religion: 
celle-ci est le premier maillon de la chaîne unissant les multiples figures de la vie collective.

L'exemple de l'évolution des peines nous l'a appris: si l'on veut identifier et analyser les maillons de la chaîne, il faut s'interroger sur des facteurs aussi bien quantitatifs que qualitatifs. On peut supposer, en effet, que, dans le processus historique, les représentations religieuses et les rituels ne sont pas seulement relégués au second rang par d'autres représentations et règles d'actions collectives, mais changent également d'un point de vue qualitatif. De fait, Durkheim voit là un processus de sécularisation de la religion; celui-ci, de son point de vue, ne se confond cependant aucunement avec une idéalisation, et pas davantage, d'autre part, avec une désacralisation. L'idéalisation est le processus qui se rattache aux représentations symboliques de tous ordres, tandis que la désacralisation affecte l'idéal collectif. De façon générale, cependant, il apparaît que tout groupe, en tant que communauté solidaire, est centré autour d'un sacré qui est particulièrement protégé. [...]

11 Durkheim présenta, principalement dans des cours, trois réflexions sur l'histoire de l'évolution qui vont nous intéresser dans ce qui va suivre: la première concerne les changements dans l'éducation, la deuxième les changements dans la pensée et la troisième les changements dans les mœurs et le droit. Chacune d'entre elles était étroitement liée au diagnostic de Durkheim sur la modernité en général et la situation française en particulier. Attardons-nous un instant sur les problèmes qui préoccupaient Durkheim à son époque.

$12 \mathrm{Au} \mathrm{XIX}$ siècle, la France connut une alternance incessante de révolutions et de restaurations. Napoléon Ier avait sonné le glas de la première république, Napoléon III celui de la seconde. La troisième était née dans l'ombre de la guerre de 1870 et de la Commune de Paris. Sur le papier, elle dura longtemps (1870-1940), mais elle ne connut guère de stabilité interne. 108 gouvernements et 561 ministres se succédèrent, même si certains furent plusieurs fois en poste. Les gouvernements restaient en fonction huit mois en moyenne. De surcroît, cette république manqua, au début du moins, de républicains convaincus. Il fallut du temps pour que, aussi bien du point de vue des idées que de celui des institutions, le républicanisme prenne le pas sur le monarchisme et la laïcité sur le cléricalisme ${ }^{16}$.

13 La Troisième République fut le cadre institutionnel dans et pour lequel Durkheim vécut. Il n'est pas exagéré de dire qu'il fut un républicain fervent et un anticlérical militant. Il considérait que son travail scientifique était placé au service de la république. Dans son optique, celle-ci avait à résoudre deux problèmes sur le mode "républicain»: la question religieuse et la question éducative. Les deux étaient à ses yeux étroitement liées. Dans les deux cas, en effet, il en allait des rapports de l'Etat français et de l'Eglise catholique : séparation de l'Eglise et de l'Etat, laïcisation de l'éducation et ces débats qui occupaient la France depuis la Révolution passionnaient Durkheim. En 1905, l'abolition du concordat mit formellement un terme à cette controverse. Les républicains radicaux qui étaient arrivés au pouvoir en 1898 et au nombre desquels figurait Durkheim avaient finalement réussi à imposer leurs vues.

La cause républicaine connut enfin une autre avancée avec l'affaire Dreyfus, qui secoua la France de 1894 à 1906. Ce fut là une conséquence que les instigateurs de l'affaire n'avaient pas prévue. Dans un premier temps, ces événements apparurent comme une grave menace pour la république. L'un des moteurs de l'affaire se révéla être l'antisémitisme, largement diffusé en France comme ailleurs. Tout commença à 
l'automne 1894, lorsqu'une femme de ménage travaillant pour le compte des services secrets français découvrit dans la corbeille à papier de l'attaché militaire de l'ambassade d'Allemagne à Paris, Maximilian von Schwartzkoppen, un avis rédigé en langue française qui annonçait la fabrication d'un nouveau modèle de canon. Ce type d'informations ne pouvait être connu que d'un membre de l'état-major de l'armée française. Le traître devait donc en faire partie. On l'identifia promptement en la personne du capitaine Dreyfus, alsacien et juif. Ces deux caractéristiques parlaient en sa défaveur. Il fut arrêté le 15 octobre 1894 - sur la base de documents falsifiés, comme on l'apprit plus tard - et condamné le 19 décembre 1894 au bannissement à vie. Sa condamnation incluait aussi la dégradation et l'exclusion de l'armée. Cette partie du jugement fut prononcée le 5 juin 1895 dans la cour de l'Ecole militaire sous la forme d'un spectacle public ; puis vint l'exil à l'Ile au Diable. Il fut révélé plus tard sans aucun doute possible qu'un innocent avait ainsi été mis au ban. Il fallut attendre 1906 pour qu'il fût officiellement réhabilité et rétabli dans son honneur. Cet événement marqua une défaite de l'alliance inique des monarchistes, des cléricaux et des militaires. Ils étaient à l'origine de la condamnation de Dreyfus et s'employèrent à provoquer de graves agressions antisémites afin d'empêcher la réhabilitation. Cependant, la république fut victorieuse. Outre la séparation de l'Eglise et de l'Etat, la subordination du pouvoir militaire au pouvoir politique fut imposée et la Ligue des Droits de l'Homme fut fondée.

Les différentes phases de l'affaire Dreyfus ne nous intéressent pas ici. Dans le long combat pour la réhabilitation du capitaine Dreyfus, un moment décisif fut la publication dans l'Aurore, le journal de Georges Clémenceau, de la fameuse lettre ouverte d'Emile Zola au président de la République, Félix Faure, sous le titre «J'accuse ! Lettre au Président de la République ». Les attaques virulentes dont Zola fit l'objet, qui l'obligèrent à fuir en Angleterre, furent à l'origine d'un mouvement de solidarisation des « intellectuels ». Durkheim s'en mêla lui aussi. Il intervint en publiant en 1898, dans La Revue bleue, alors que l'affaire battait son plein, un article intitulé « L'individualisme et les intellectuels » : à ce moment, l'innocence de Dreyfus ne faisait plus de doute pour tous ceux qui examinaient sans préjugés les faits connus. Dans cet article, Durkheim défend l'individualisme moral, dans la lignée de Rousseau et de Kant et l'oppose d'un côté à l'individualisme égoïste des économistes, d'un côté, et, de l'autre, à l'antiindividualisme des forces conservatrices qui jouèrent un si grand rôle dans l'Affaire Dreyfus.

16 Le primat de l'individualisme égoïste était aussi, pour Durkheim, le problème de la société moderne en tant que telle. En effet, comme il l'avait déjà montré dans l'étude sur la division du travail, celle-ci prônait une forme d'individualisme associant autonomie personnelle et attachement au groupe. Lorsque cet individualisme recevait une interprétation strictement utilitariste et se trouvait institutionnalisé par le biais du marché anonyme, il en résultait la division des membres de la société et une désintégration de la société moderne. L'état d'anomie menaçait alors de s'installer, même si la prospérité allait croissant ${ }^{17}$. La position de Durkheim, qui n'a été esquissée ici qu'à grands traits, a été décrite comme une défense communautariste du libéralisme. Nous le verrons, cette définition va effectivement à l'essentiel ${ }^{18}$.

Il faut ici mentionner un autre facteur, avant de revenir sur les trois lignes d'évolution retracées par Durkheim. Ce dernier ne voyait pas seulement dans la science nouvelle qu'était la sociologie un outil de diagnostic, mais aussi de thérapie. D'où l'intérêt 
particulier qu'il portait à l'institutionnalisation de cette discipline dans le système éducatif français, dans les universités notamment et à l'intégration, dans les différents cursus d'enseignement, des connaissances sociologiques relatives aux causes et aux modes de fonctionnement de la vie collective. On pourrait même dire qu'il chercha à fonder une école, faisant preuve d'un sens de la stratégie tout à fait accusé. Comme il n'était pas aisé de placer les sociologues dans les universités (dans un premier temps lui-même n'était parvenu à obtenir, non sans difficultés, qu'un poste de professeur de pédagogie à la Sorbonne, qui ne fut transformé en chaire de pédagogie et de sociologie qu'en 1913), Durkheim chercha à diffuser ses idées dans d'autres disciplines. Le relais le plus important, de ce point de vue, fut L'Année sociologique. Avec l'aide de son neveu Marcel Mauss, Durkheim en fit un forum de publications de son courant scientifique. Dans un premier temps, elle comprenait deux parties : les « Mémoires originaux » (des essais) et les "Notes critiques" (des comptes rendus ${ }^{19}$ ). Ces dernières portaient sur les principales publications de l'année précédente en français, anglais, allemand ou italien ${ }^{20}$. L'Année sociologique devint rapidement une revue critique couvrant un spectre de thématiques très large, dont le rayonnement international était considérable. Durkheim commandait des comptes rendus de tout ce qui pouvait entrer dans sa large définition des faits sociaux. Il n'hésita pas à faire appel à des représentants d'autres disciplines, surtout à des étudiants. Il eut aussi souvent recours à ce forum pour diffuser ses propres travaux : ses contributions aux douze volumes (articles et comptes rendus) parus entre 1896/1897 et 1912 avoisinent les 700 pages $^{21}$. Ses comptes rendus personnels portaient surtout sur des ouvrages allemands. Mais il est un fait plus décisif encore: il tissa de cette façon un réseau scientifique ${ }^{22}$. Son ambition, dans cette entreprise, était d'imposer la sociologie dans le concert des disciplines scientifiques et, d'autre part, dans les programmes pédagogiques au sein et en dehors des universités.

Ceci nous amène au premier texte que nous allons examiner brièvement dans une perspective d'histoire de l'évolution. Il est tiré d'un cours sur "L'Education morale » que Durkheim avait donné en 1902/1903 à la Sorbonne, où il venait d'être nommé à une chaire de pédagogie ${ }^{23}$. L'objectif de ce cours était de défendre l'idée que l'école, autrement dit le système éducatif public, était le lieu institutionnel qualifié pour l'éducation morale, et d'inciter à faire commencer celle-ci dès l'école primaire, afin que la morale affective et personnelle de la famille trouve un complément et un élargissement dans une morale cognitive et impersonnelle. Il s'agissait également de fonder cette éducation morale sur le principe de "laïcité », autrement dit d'assumer de façon conséquente la sécularisation de la morale. La Sorbonne - on parlait de la "Nouvelle Sorbonne» et ses adversaires brocardaient même une Sorbonne " germanisée » - était devenue le foyer du républicanisme et du sécularisme, et prônait un nouvel idéal éducatif dans lequel la science, y compris la science de la morale, devait se substituer à la littérature ${ }^{24}$. Le cours était composé dans cet esprit. Il comprenait deux parties, une théorique et l'autre pratique. Le tout était précédé d'une introduction à la morale laïque. Durkheim voyait en elle le noyau de la grande révolution pédagogique amorcée en France depuis une vingtaine d'années. Elle avait pour but de " donner à nos enfants, dans nos écoles, une éducation morale qui fût purement laïque ", " une éducation purement rationaliste », qui «s'interdise tout emprunt aux principes sur lesquels reposent les religions révélées ${ }^{25}$ ».

19 Nous ne nous intéresserons pas ici à la distinction que Durkheim propose de faire entre science et pédagogie, ni à la partie pratique du cours, bien qu'il soit important de noter que Durkheim attribuait aux sciences naturelles une portée pédagogique majeure. Rien 
de surprenant à cela : cette orientation s'explique aussi bien par le nouvel idéal éducatif défendu par le sociologue que par le malaise suscité chez lui par la formation qu'il avait reçue à l'Ecole normale supérieure. Il reprochait aux enseignements dispensés à l'Ecole d'être trop littéraires et pas assez scientifiques. Il voulait changer les choses: son objectif n'était pas la transmission des lettres mais de la science positive. Nous nous arrêterons brièvement sur la partie théorique en la rattachant aux observations que nous avons faites au sujet de la définition et de l'explication des faits moraux. Le cours sur l'éducation morale complète les considérations précédentes en les abordant du point de vue mental.

En effet, comme on pouvait s'y attendre dans un tel cours, le problème de la morale est essentiellement abordé du point de vue de l'élève en voie de socialisation. La question est la suivante : comment l'enfant peut-il devenir un membre à part entière de la société, capable d'actions morales? Durkheim considère que trois compétences sont nécessaires à cette fin : l'enfant doit apprendre à se discipliner, il doit développer la capacité à assumer son appartenance à des groupes multiples et donc à exercer différentes formes de solidarité, et il doit pouvoir être autonome. Discipline de soi, attachement à des groupes multiples et autonomie, telles sont, pour lui, dans la société moderne, les caractéristiques essentielles d'une personne capable d'actions morales. $\mathrm{Si}$ l'on cherche une comparaison historique, on doit donc observer quel type de morale favorise, chez l'élève, quel esprit de discipline, quel attachement au groupe social et quelle détermination de la volonté. Ces trois éléments - esprit de discipline, lien au groupe social et détermination de la volonté - sont ainsi au centre des comparaisons.

Durkheim oppose alors deux configurations historiques et, en même temps, il s'efforce de les replacer dans la perspective d'une histoire de l'évolution. La formule est la suivante : de la morale religieuse à la morale laïque. Il ne s'agit pas d'affirmer que la morale laïque est affranchie de tout vestige de religion. Durkheim ne le prétend pas, on l'a vu plus haut. Mais, pour lui, que l'esprit de discipline revête la forme d'une pure contrainte extérieure ou d'une contrainte sur soi-même, que l'attachement au groupe social soit seulement prescrit ou assumé personnellement, que la volonté soit déterminée de l'extérieur, par la croyance dans une autorité voulue par Dieu, ou qu'elle se détermine elle-même par la raison et des idées éclairées, ne revient en aucun cas au même. A chaque fois, la première partie de l'alternative est le propre de la morale religieuse, la seconde de la morale laïque. Ces deux morales créent des personnes morales différentes; on pourrait dire, avec David Riesman, que les premières sont davantage régies de l'extérieur, les secondes de l'intérieur. Durkheim parle lui-même de morale hétéronome et de morale autonome.

La première question qui se pose est la suivante: à quel niveau et à quel type d'évolution ces distinctions s'appliquent-elles? Au niveau individuel ou au niveau collectif? A l'évolution ontogénétique ou à l'évolution phylogénétique ? La réponse ne peut être que celle-ci : aux deux à la fois. Mais c'est justement ce qui pose problème. Si l'on se place au niveau collectif et que l'on considère l'évolution phylogénétique, on a affaire à deux types de morale sociale qui correspondent à chaque fois à une structure sociale et à une forme de solidarité différentes : le collectivisme moral, associé à la société segmentaire et à la solidarité mécanique, d'une part, et, d'autre part, l'individualisme moral, associé à la société de la division du travail et à la solidarité organique. Si l'on se place au niveau individuel et que l'on considère l'évolution ontogénétique, on a affaire à deux stades de la morale enfantine. Leur développement 
semble tributaire d'appartenances de groupe. On pense à George Herbert Mead : ce qui compte, c'est le type et le nombre « d'autres".

Nous l'avons vu dans l'étude des faits moraux : il faut établir une distinction entre la dimension de la règle et celle de la motivation. A l'instar de Kant, Durkheim s'intéresse à la fois à des règles et à des mobiles, et au lien entre les unes et les autres. La dimension de la règle recouvre, chez lui, l'idée de devoir et, en même temps, l'idée du bien, qui forment ensemble la morale objective et ne peuvent être séparées l'une de l'autre; la dimension de motivation recouvre quant à elle le sentiment ambivalent qui mêle le respect et l'amour, tous deux constituant la morale subjective ou, en d'autres termes, l'attitude morale ou la mentalité. L'individu peut, en effet, selon Durkheim, être plus fortement marqué par l'idée du devoir ou par l'idée du bien. La première option correspond à une attitude de froide maîtrise de soi, la seconde à des élans spontanés ${ }^{26}$. L'individu a des devoirs vis-à-vis des groupes auxquels il appartient, et ceux-ci lui imposent des limites. Dans le même temps, cependant, ils représentent le bien auquel on tient pour digne d'aspirer et, par là, ils permettent à l'individu de se dépasser. Ils l'autorisent à se civiliser, ou, en d'autres termes, à se moraliser et à se cultiver. Ce constat s'applique à toute morale objective. La spécificité de la morale laïque, selon Durkheim, est cependant de ne pas en rester là. Bien entendu, les devoirs particuliers envers le groupe doivent être remplis, mais la morale laïque impose également de remplir des devoirs universels envers l'humanité. Pour Durkheim, c'est là le résultat d'un long processus qui, dans l'histoire de la civilisation, a connu son point culminant avec la Révolution française. L'individualisme moral est, par suite, orienté vers ces deux objectifs : la préservation et l'extension des groupes concrets mais aussi de l'humanité abstraite, sous la forme de l'homme idéal, la " personne humaine ${ }^{27}$ ».

$24 \mathrm{Au}$ demeurant, si pertinentes que ces distinctions puissent paraître au premier abord, une question demeure : comment comprendre la succession et les relations mutuelles de ces différents moments? Cette question conduit à s'intéresser de plus près à la notion d'autonomie chez Durkheim. Dans ce contexte, en effet, il parle d' "autonomie progressive » ou d'autonomisation ${ }^{28}$. Que faut-il entendre par là ?

Pour répondre à cette question, nous partirons d'un autre point de vue avant de revenir à Durkheim. Que signifie l'autonomie en psychologie et en philosophie ? Ou plutôt : que peut signifier l'autonomie ici ? Pour la psychologie, nous nous réfèrerons à Jean Piaget ; pour la philosophie, à Emmanuel Kant.

Dans la première phase de son travail, Piaget consacra au jugement moral de l'enfant une étude fondée sur quantité d'observations, dans laquelle il évoquait aussi la sociologie de l'éducation de Durkheim. Dès le début de cette étude, il formule le problème qui nous occupe: "Toute morale consiste en un système de règles et l'essence de toute moralité est à chercher dans le respect que l'individu acquiert pour ces règles ${ }^{29}$ ». D'où un tel respect provient-il ? C'était déjà la question soulevée par Kant. Dans le cas présent, elle est posée du point de vue de la psychologie de l'enfant.

Piaget étudia des enfants âgés de 4 à 13 ans en se demandant comment la conscience morale se constituait et se développait chez ces derniers. Pour ce faire, il eut recours à des règles de jeu: en l'occurrence, celles du jeu de billes. On peut évidemment se demander si les règles de ce type sont adaptées au problème qui nous occupe. Je laisse cette question de côté. Ce qui est décisif, de notre point de vue, c'est que Piaget découvre un lien entre les formes de coopération et la conscience des règles. Il en résulte une distinction entre deux types de morale. Le premier type représente une 
morale de la contrainte, dans laquelle le respect unilatéral, la justice du « œil pour œil, dent pour dent", la responsabilité objective et la contrainte sociale sont prédominants; la seconde est une morale de la coopération, fondée sur le respect mutuel, la justice distributive, la responsabilité subjective et la collaboration sociale. On peut désigner la première comme hétéronome, la seconde comme autonome. Elles se présentent successivement. L'enfant se développe et développe aussi pas à pas une morale autonome. Les découvertes qui amenèrent Piaget à cette conclusion furent plus tard reprises par Lawrence Kohlberg et intégrées dans un schéma plus détaillé des étapes du jugement moral. Kohlberg ne parle plus de morale autonome et hétéronome, mais de niveau de jugement préconventionnel, conventionnel et postconventionnel. Chaque niveau comprend deux sous-niveaux. Rapportés à cette série, les deux stades du jugement moral observés par Piaget chez l'enfant se situent au niveau préconventionnel. Kohlberg fut explicite sur ce point ${ }^{30}$.

Les résultats initiaux obtenus par Piaget sont résumés dans la synthèse 1.

Chez Kant, la notion d'autonomie est inséparable de l'idée de liberté, dont il dit qu'elle ne peut pas être démontrée mais qu'elle doit être défendue comme un présupposé nécessaire si nous cherchons à comprendre pourquoi nous pouvons être rendus responsables de nos actions. L'autonomie de la volonté, pour lui, est en même temps le principe suprême de notre action morale. En effet, il ne signifie pas autre chose que ceci : choisir une action de telle sorte que «je puisse aussi vouloir que ma maxime devienne une loi universelle ${ }^{31} »$. L'autonomie de la volonté implique celle d'un être raisonnable, qui satisfait aux exigences d'universalité sur lesquelles tous les êtres raisonnables doivent s'accorder. Une morale hétéronome ne se fonderait pas sur une loi universelle et, au sens strict, ne serait en aucune manière une morale. Pour Kant, morale et autonomie vont de pair. Ce qui revient à dire que celui qui remplit les commandements de la morale, et fait donc son devoir, n'est «soumis qu'à sa propre législation, encore que cette législation soit universell ${ }^{32} »$.

Synthèse 1 : Systématisation des résultats obtenus par Piaget

\begin{tabular}{|c|c|c|c|}
\hline Relation sociale & Application de la règle & Conscience de la règle & Notion de la justice \\
\hline \multirow[t]{3}{*}{ Pas de coopération } & \multirow[t]{3}{*}{ Chacun joue pour soi } & \multirow[t]{2}{*}{ Pas de conscience de la règle } & $\begin{array}{l}\text { Le droit est ce qui est fixé par } \\
\text { les adultes }\end{array}$ \\
\hline & & & Justice immanente \\
\hline & & Pas de respect & L'autorité prime sur l'égalité \\
\hline \multirow[t]{3}{*}{ Début de coopération } & \multirow{3}{*}{$\begin{array}{l}\text { On commence à jouer avec } \\
\text { d'autres, mais on veut toujours } \\
\text { gagner }\end{array}$} & Conscience de la règle & \multirow{2}{*}{$\begin{array}{l}\text { Le droit est ce qui satisfait aux } \\
\text { principes d'égalité. } \\
\text { Justice distributive }\end{array}$} \\
\hline & & $\begin{array}{l}\text { Les règles sont considérées comme } \\
\text { sacrées et intangibles }\end{array}$ & \\
\hline & & Respect unilatéral & L'égalité prime sur l'autorité. \\
\hline \multirow[t]{3}{*}{$\begin{array}{l}\text { Coopération } \\
\text { développée }\end{array}$} & \multirow{3}{*}{$\begin{array}{l}\text { Tous jouent ensemble et s'en } \\
\text { tiennent aux règles connues de } \\
\text { tous et reconnues par tous }\end{array}$} & Conscience de la règle & $\begin{array}{l}\text { Le droit est ce qui satisfait aux } \\
\text { principes de l'équité }\end{array}$ \\
\hline & & $\begin{array}{l}\text { Les règles reposent sur un consen- } \\
\text { sus et on est tenu de s'y conformer }\end{array}$ & Justice compensatoire \\
\hline & & Respect mutuel & L'équité prime sur l'autorité \\
\hline
\end{tabular}

Discutant le concept d'autonomie, Durkheim ne suit ni la définition de Piaget, ni celle de Kant. Commençons par Kant. Les grandes lignes de la critique durkheimienne de Kant ont déjà été évoquées. On la retrouve ici, sous une forme plus radicale, appliquée au problème de l'autonomie. Durkheim cite notamment le début du chapitre 8 de la Critique de la raison pratique: "L'autonomie de la volonté, dit-il, est l'unique principe de toutes les lois morales et de tous les devoirs qui y sont conformes : toute hétéronomie de 
la volonté ... est opposée ... à la moralité de la volonté ${ }^{33}$ ». L'antagonisme ainsi relevé entre autonomie et hétéronomie est pour Durkheim la pierre d'achoppement du raisonnement kantien. Selon lui, en effet, cette construction ne peut fonctionner que si l'on postule une distinction entre monde intelligible et monde sensible. Or, pour Durkheim, celle-ci est inadmissible. Kant «dénaturalise " la sphère intelligible alors qu'elle fait partie de la nature. En effet, notre raison est aussi « naturelle » que notre sensibilité. L'autonomie et l'hétéronomie ne sont antinomiques qu'en apparence, tout comme le devoir et le bien, la limitation et l'extension, l'individu et le groupe. Partout, il s'agit des deux faces d'une même médaille, d'un « mélange » (conflictuel il est vrai) de composantes qui, en fin de compte, forment pourtant une unité parce qu'elles font partie de notre nature. Il est certes très important d'observer les tensions qui en résultent, mais elles ne procèdent en rien d'un dualisme entre monde nouménal et monde phénoménal. Ce dualisme n'est qu'une réponse métaphysique à une question qui doit recevoir une réponse empirique. Si la réponse métaphysique était juste, il ne serait pas nécessaire que le devoir moral possédât un caractère impératif et obligatoire : il serait « accidentel ». En effet, le pouvoir contraignant du devoir moral ne se manifesterait alors qu'en cas de conflit entre la raison et les passions ${ }^{34}$.

L'argument de Durkheim est déroutant : Kant souligne lui-même expressément que la raison doit nécessairement entrer en conflit avec les passions parce que l'homme n'est pas seulement un être rationnel mais aussi un être naturel. Un pur être de raison ne serait, de fait, pas contraint de suivre la loi pratique. Pour Kant, il ne peut fondamentalement pas exister de situation dans laquelle nous ne soyons pas contraints dans notre action, que celle-ci soit accomplie conformément au devoir ou par devoir. Une telle action présuppose toujours, chez lui, le dépassement de soi. Par conséquent, la contrainte n'est pas "accidentelle » comme le pense Durkheim : c'est une donnée indissociable de la double nature de l'homme et une conséquence du fait que, en tant qu'êtres rationnels, nous sommes aussi des êtres naturels.

Durkheim ne souhaite pas, au demeurant, bannir le dualisme kantien, mais seulement le naturaliser et le sociologiser. En effet, " notre nature toute entière a besoin d'être limitée, contenue, bornée ; notre raison, tout aussi bien que notre sensibilité ${ }^{35} »$. En d'autres termes: au sens strict, il nous est tout simplement impossible de devenir autonomes. En effet, l'hétéronomie est constitutive de notre existence individuelle. Si cette limitation extérieure n'existait pas, nous nous détruirions nous-mêmes. Par conséquent, la morale à laquelle nous nous plions de façon autonome nous est en même temps toujours imposée. Il s'agit d'une morale contraignante qui nous limite et constitue une « barrière idéale ${ }^{36}$ » contre nos passions. Dans ce cas, l'autonomie signifie seulement que nous comprenons pourquoi cette limitation imposée est nécessaire. L'instance qui nous permet de le comprendre n'est cependant pas une révélation religieuse. C'est la science de la morale positive. A l'aide de cette dernière, nous pouvons connaître scientifiquement les «lois» ordonnées par la morale. Et dans la mesure où nous les connaissons, les exigences morales qui nous sont imposées reçoivent notre « consentement éclairé37 ".

Si nous abordons maintenant Durkheim à partir de Piaget, il apparaît d'emblée que celui-ci, du point de vue de Piaget, n'est pas en mesure, en fin de compte, de distinguer contrainte et collaboration, morale de la contrainte et morale de la coopération, bien que ces oppositions s'accordent avec l'argumentation de La Division du travail ${ }^{38}$. Si nous nous référons également à Kohlberg, ce que Durkheim appelle la morale autonome se 
situe tout au plus, sur le plan ontogénétique, au niveau des jugements moraux conventionnels. Kohlberg définit le degré 4 du niveau conventionnel comme suit : la personne qui juge adopte le point de vue de la société et tient pour légitime de remplir les devoirs traditionnels et de se conformer aux lois afin de contribuer au bon fonctionnement de la société et de préserver celle-ci de la faillite ${ }^{39}$. L'individu s'adapte donc par conviction aux attentes sociales. Mais c'est exactement ainsi que Durkheim décrit le comportement des personnes qui ont une action morale autonome au sens où il l'entend. En effet, on l'a dit, c'est par « consentement éclairé » qu'ils se conforment à la morale que requiert la société de la division du travail : «A condition de posséder une intelligence adéquate des préceptes moraux, des causes dont ils dépendent, des fonctions que chacun d'eux remplit, nous sommes en état de ne nous y conformer qu'à bon escient et en connaissance de cause. Un conformisme ainsi consenti n'a plus rien de contraint ${ }^{40} »$.

Toutefois, pour Durkheim, la morale requise par la société de la division du travail n'est pas la morale religieuse mais la morale laïque. Si on la compare avec la morale religieuse, elle possède donc une autre structure. Elle exige de l'individu qu'il ne remplisse pas seulement les devoirs particuliers d'un groupe, mais aussi des devoirs universels de l'humanité. Elle réclame donc, de fait, une capacité d'autodétermination accrue, autrement dit une capacité d'autonomie. Les analyses situées sur le plan collectif et individuel, ontogénétique et phylogénétique, ne s'accordent donc pas bien entre elles. Cela tient manifestement au fait que Durkheim réduit la notion d'autonomie à la compréhension de la nécessité de se soumettre aux exigences de la société. A proprement parler, il ne peut exister pour Durkheim d'autonomie régie par des principes mais consciente de sa liberté par rapport à l'exigence de soumission aux normes sociales. C'est là le résultat, d'une part, de la sociologisation et de la naturalisation de Kant, et, d'autre part, du fait que Durkheim, parce qu'il défend une théorie sociologique holiste, est contraint de rejeter le modèle interactionniste dont Piaget pose les fondements.

Pour sa défense, on pourrait cependant dire que Durkheim ne fait là que tirer les conséquences des thèses défendues dans $L a$ Division du travail: l'autonomie et la dépendance ne s'annulent pas l'une l'autre; davantage d'autonomie va de pair avec davantage de dépendance. Durkheim ne nie pas non plus que l'exigence d'autonomie progressive soit, à l'évidence, un signe des temps, et que la participation active de l'individu à la vie morale soit amenée à se développer au fur et à mesure que le type de structure segmentaire laisse la place à celle qui est associée à la division du travail. Cependant, tout cela ne change rien au primat de la vie collective sur la vie individuelle. Même l'individualisme est un idéal collectif qui soumet l'individu à une contrainte collective. Si éclairé que soit notre consentement, le collectif nous limite, tout en nous contraignant à nous dépasser et à faire quelque chose que nous ne ferions pas en l'absence de cette contrainte. Par suite, nous gardons toujours un sentiment d'obligation imposée. Rares sont, chez Durkheim, les moments où l'individu cesse d'éprouver ce sentiment. C'est le cas des phases de créativité collective. Dans les sociétés segmentaires, ce sont notamment les cérémonies religieuses qui ont pour fonction de les déclencher; dans les sociétés modernes, ce sont surtout les révolutions. Cependant, dès que le quotidien reprend le dessus, le sentiment de fusion avec le groupe disparaît et le sentiment d'obligation redevient prédominant. 
Là où Kant place le sujet transcendantal, Durkheim lui substitue la société. Mais ce qui a été dit de la morale vaut aussi pour la pensée. Si nos actions morales autonomes ellesmêmes sont accompagnées d'un sentiment de contrainte, on peut en dire autant de l'usage éclairé de notre raison. Les notions auxquelles nous avons recours pour ordonner la réalité sont elles aussi imposées par le collectif. Cela vaut pour les sociétés simples, comme le montrait l'étude sur les formes primitives de classification, mais cela ne cesse pas d'être vrai aujourd'hui, en un temps où la science moderne est florissante. En effet, notre raison n'est pas « une faculté transcendante : elle fait partie du monde et, par conséquent, elle subit la loi du monde ${ }^{41} »$. Or ces lois sont des lois de limitation par le collectif, mais il est vrai qu'elles rendent aussi possible la pensée et l'action individuelles.

Durkheim dit expressément que la morale laïque possède une composante cognitive. Pour lui, c'est une morale scientifique ou, du moins, une morale qui porte la marque de la science. Il semblerait même, au premier abord, que ce soit cette composante cognitive qui distingue la morale laïque de la morale religieuse. En effet, l'argumentation de Durkheim semble présupposer que seule la morale laïque associe à l'esprit de discipline et à l'attachement au groupe social un troisième élément, la détermination de la volonté qui justifie la morale. Cependant, cette hypothèse n'est guère plausible car, du point de vue de Durkheim, le totémisme associe déjà un système de comportement et un système de pensée. L'idéal collectif englobe toujours les deux : une "prescription" relative à la manière dont la réalité doit être pensée, et une prescription relative à la manière dont on doit agir dans la réalité.

Lorsqu'on compare les deux morales, la question n'est donc pas de savoir si l'une, à la différence de l'autre, possède une composante cognitive, mais seulement de savoir en quoi elle consiste. Ceci nous ramène à l'étude sur les formes primitives de classifications. En effet, les classifications primitives dérivent, selon Durkheim, d'une pensée mythologique. Si la formule de l'évolution de la morale est « de la morale religieuse à la morale laïque ", la formule de l'évolution de la pensée est « de la pensée mythologique à la pensée scientifique ».

Nous en arrivons ainsi au deuxième cours que nous souhaitons prendre en considération, le cours sur l'évolution de la pensée. Durkheim le présenta à la Sorbonne en 1913/1914, en faisant la synthèse de beaucoup de recherches antérieures. Il fut ensuite reconstitué sur la base de notes d'étudiants et publié à titre posthume sous le titre « Pragmatisme et sociologie ${ }^{42} »$.

Avec un pareil titre, on pourrait s'attendre à ce que Durkheim, le critique de Kant, entreprenne de refonder philosophiquement la sociologie à partir du pragmatisme, comme cela a pu être fait aux Etats-Unis, notamment dans l'Ecole de Chicago. Mais tel n'est aucunement le cas : Durkheim oppose la sociologie au pragmatisme. Ce dernier est pour lui un utilitarisme logique et n'est pas en mesure d'expliquer ce dont Kant avait fourni une explication, bien que celle-ci soit restée prise dans la métaphysique: comment il peut exister des jugements de connaissance universels et nécessaires, dotés d'une force contraignante. A la place du pragmatisme, mais bien entendu aussi à la place de Kant, Durkheim prône, on l'a dit, une sociologie de la connaissance qui dépasse aussi bien le pragmatisme que le rationalisme traditionnel d'une philosophie grevée de métaphysique.

Dès lors, pourquoi Durkheim se réfère-t-il au pragmatisme? Pourquoi s'intéresse-t-il à ce courant philosophique? La raison en est la suivante : le pragmatisme a eu le mérite 
de s'attaquer au rationalisme qui a marqué la tradition philosophique dans son ensemble et la culture française en particulier. Il a réveillé la pensée philosophique de son sommeil dogmatique ${ }^{43}$ et révèle les faiblesses dont souffre le rationalisme philosophique traditionnel. C'est en ce sens que la critique pragmatique du rationalisme présente un intérêt particulier, du point de vue de Durkheim. Cependant, elle lui sert seulement de point de départ pour exposer sa propre position.

Dans ce cours sont associées deux lignes d'argumentation que nous allons dissocier pour les besoins de notre exposé. La première concerne l'évolution de la pensée, qui se résume, nous l'avons vu, dans la formule «de la pensée mythologique à la pensée scientifique ». La seconde concerne le rapport de la sociologie de la connaissance au pragmatisme et au rationalisme traditionnel. La première ligne argumentative se rattache à la discussion sur l'évolution de la morale et la prolonge; la seconde nous ramène à notre discussion sur les Règles de la méthode sociologique.

La première ligne argumentative n'est pas longue à retracer. Comme pour la morale et la structure, Durkheim distingue ici deux types : la mythologie et la science ${ }^{44}$. On peut distinguer l'une et l'autre à partir de l'idée de vérité, de l'idée de réalité et d'ordre des concepts, autrement dit du principe de classification dominant. [...] Pour Durkheim, c'est Aristote qui marque un tournant dans l'évolution de la pensée logique. Chez lui, le principe de transmutation est enfin remplacé par le principe de séparation logique, l'organisation des concepts selon le schéma genus proximum, differentia specifica.

Cette typologie peut se présenter ainsi :

Synthèse 2 : Evolution de la pensée

\begin{tabular}{lll}
\hline & Pensée mythologique & Pensée scientifique \\
\hline Idée de la vérité & $\begin{array}{l}\text { Dogmatique } \\
\text { Pas d'examen méthodique empirique }\end{array}$ & $\begin{array}{l}\text { Critique } \\
\text { Fondée sur un examen méthodique } \\
\text { empirique }\end{array}$ \\
Idée de la réalité & $\begin{array}{l}\text { Symbolique } \\
\text { "Subjective » }\end{array}$ & $\begin{array}{l}\text { Réaliste } \\
\text { "Objective " }\end{array}$ \\
Principe de classification & $\begin{array}{l}\text { Principe de transmutation } \\
\text { Hiérarchies de concepts flexibles } \\
\text { La réalité est divisée en niveaux } \\
\text { distincts mais continus. }\end{array}$ & $\begin{array}{l}\text { Principe de séparation } \\
\text { Hiérarchies de concepts logiques }\end{array}$ \\
\hline
\end{tabular}

Etant donné que toute morale possède une composante cognitive, nous pouvons rapprocher les réflexions de Durkheim sur l'évolution de la pensée de ses réflexions sur l'évolution de la morale. Ce faisant, il faut tenir compte de la distinction relevée plus haut entre la dimension de la règle et celle de la motivation. A quoi s'ajoute celle de la sanction [...]. Pour finir, il faut également prendre en considération, dans cette vue d'ensemble synthétique, les différents types d'écart [...] (cf. synthèse 3).

La deuxième ligne argumentative est moins facile à suivre. La référence à Aristote ne suffit pas, bien entendu, à retracer l'histoire complexe du rationalisme philosophique. Dans son cours, Durkheim ne cherche pas à entrer dans des distinctions plus précises. L'adversaire majeur est la tradition du rationalisme philosophique dans son ensemble, d'Aristote à Kant, Hegel et au-delà. Le pragmatisme, pour Durkheim, est le représentant d'une pensée postmétaphysique, et il lui sert en premier lieu à revendiquer la nécessité 
d'un renouveaudu rationalisme traditionnel, que sa sociologie de la connaissance a pour but de rendre possible.

Nous ne débattrons pas ici de la pertinence de l'approche durkheimienne du mouvement pragmatiste. Il ne fait pas de doute qu'elle est insuffisante ${ }^{45}$. Durkheim rend hommage à la critique pragmatiste du rationalisme philosophique traditionnel, mais tient sa solution du problème de la vérité pour infondée: notre pensée, le pragmatisme a raison de le souligner, ne consiste pas à reproduire (que ce soit de façon matérielle ou idéelle) mais à produire des développements nouveaux; cependant, elle ne le fait pas seulement pour des raisons utilitaires. Notre pensée et notre action, comme le pragmatisme le souligne à juste titre, sont liées entre elles, mais un fossé les sépare cependant. En fin de compte, Durkheim oppose son concept de la vérité à celui du pragmatisme. Il met en avant quatre traits caractéristiques : la vérité est attachée à l'obligation morale de l'individu qui doit aspirer à la vérité ; la vérité est contraignante pour l'individu, elle doit être reconnue par lui ; la vérité est impersonnelle, elle vaut donc pour tous ; et la vérité est également spéculative, car elle est pour une part une fin en soi, à laquelle on aspire pour elle-même. On le voit : la vérité est pour Durkheim un fait social, extérieur à l'individu et contraignant pour lui, universel et indépendant de lui. Comme pour la morale, Durkheim conteste, en ce qui concerne la pensée, la justesse de l'interprétation utilitariste.

Synthèse 3 : Evolution de la morale et de la pensée

\begin{tabular}{|c|c|c|c|c|}
\hline Aspect de la règle & Morale religieuse (hétéronome) & Morale lä̈que (autonome) & Ecart & \\
\hline Idée du devoir & Obligations de groupe particulières & $\begin{array}{l}\text { Obligations humaines universelles et } \\
\text { obligations de groupe particulières }\end{array}$ & Fatalisme & Anomie \\
\hline \multirow[t]{2}{*}{ Idée du bien } & Collectivisme moral, & Individualisme moral, & Altruisme & Egoïsme \\
\hline & $\begin{array}{l}\text { Préservation et extension des } \\
\text { groupes concrets }\end{array}$ & $\begin{array}{l}\text { Préservation et extension de } \\
\text { l'humanité abstraite et des groupes } \\
\text { concrets }\end{array}$ & & \\
\hline Idée de la justification & Croyance - pensée mythologique & Raison - pensée scientifique & & \\
\hline Aspect de motivation & Morale religieuse (hétéronome) & Morale laïque (autonomie) & Sentiments d'écart & \\
\hline Sentiment de respect & Respect à l'égard de l'autorité concrète & Respect à l'égard de l'idée abstraite & Désespoir & Agression \\
\hline Sentiment d'amour & Amour du membre de groupe idéal & Amour de l'homme idéal & Enthousiasme & Apathie \\
\hline Mentalité & Gouverné de l'extérieur - hétéronome & Gouverné de l'intérieur - autonome & & \\
\hline Aspect de sanction & Morale religieuse (hétéronome) & Morale laïque (autonome) & & \\
\hline Réaction & Principalement répressive & Principalement restitutive et appellative & & \\
\hline Contrôle & Principalement contrôle de l'extérieur & Principalement contrôle de soi & & \\
\hline Peine & Peine physique & Peine psychique & & \\
\hline
\end{tabular}

Durkheim associe cette idée de vérité avec des stades de connaissance correspondant aux niveaux d'émergence déjà énumérés plus haut : au niveau "inférieur ", enracinée dans l'organisme, la perception sensible encore attachée à l'instinct; au niveau "supérieur ", les images de la représentation, ancrées dans la conscience individuelle, qui expriment en même temps des tendances de l'action ; au niveau «le plus élevé ", les constructions conceptuelles qui, pour une large part coupées de l'action, sont rattachées à la conscience collective ${ }^{46}$. Ceci nous ramène à la distinction de la pensée mythologique et de la pensée scientifique. Seule la pensée scientifique sous sa forme la plus avancée, c'est-à-dire postmétaphysique et sociologique, est capable de formuler un tel concept de la vérité. Le rationalisme positiviste des règles a fait place à un rationalisme sociologique. Par là, explique Durkheim, il prolonge la tradition 
philosophique du rationalisme occidental et de la culture française en la renouvelant (cf. synthèse 4).

C'est dans une morale laïque qu'il faut chercher le pendant de cette pensée scientifique. Nous avons déjà relevé ce lien. Il est vrai que nous n'avons décrit cette morale laïque que schématiquement. Son architecture spécifique n'a été évoquée qu'en marge de notre exposé. Ceci nous amène au troisième cours dont il va être question ici. Durkheim fit allusion à ce problème devant les membres de la Société française de philosophie dans un exposé sur la détermination du fait moral. C'est le cours sur la psychologie des peuples théorique et appliquée, qu'il donna plusieurs fois, d'abord entre 1890 et 1900 à Bordeaux, puis entre 1904 et 1912 à Paris. Il porte le titre Leçons de sociologie. Physique des mours et du droit et a été transcrit par Durkheim lui-même sous le titre Physique des mours et du droit, mais n'est paru qu'à titre posthume.

Ne serait-ce qu'en raison de son titre, ce cours ne peut manquer d'évoquer Kant. Comment ne pas penser à la Métaphysique des mours et à ses deux parties, les Premiers principes métaphysiques de la doctrine du droit et les Premiers principes métaphysiques de la doctrine de la vertu? Comme Kant, Durkheim s'intéresse au droit et à la morale et il choisit un plan dans lequel les considérations sur les faits juridiques débutent par le droit privé, avant de passer au droit public; pour ce qui est des faits moraux, il se conforme à la distinction entre les devoirs envers soi-même et les devoirs envers autrui. Il est vrai, nous l'avons déjà montré, que Durkheim ne se conforme pas de manière systématique à la distinction kantienne entre les devoirs du droit et les devoirs de la vertu, entre l'ordre de la liberté extérieure et l'ordre de la liberté intérieure, et ne reprend pas non plus exactement à son compte les positions de Kant sur les rapports que les uns et les autres entretiennent entre eux. Son critère de définition pour les faits juridiques et moraux réside dans les sanctions ${ }^{47}$, alors que Kant se demande quand la liberté de l'individu peut s'accorder avec celle d'autrui en fonction d'une loi universelle, et à quelles conditions la loi que l'on se donne à soi-même peut être, en même temps, une loi valable universellement.

Synthèse 4 : Positions fondamentales en matière de théorie de la connaissance

\begin{tabular}{|c|c|c|}
\hline Rationalisme classique ou traditionnel & Pragmatisme & Sociologie \\
\hline Anhistorique et individualiste & Historique et individualiste & Historique et collectiviste \\
\hline Pensée action & Action pensée & Action pensée \\
\hline $\begin{array}{l}\text { Jugement de connaissance nécessaire et } \\
\text { absolu en raison de la nature éternelle de } \\
\text { la raison }\end{array}$ & $\begin{array}{l}\text { Jugement de connaissance conditionné } \\
\text { et relatif en fonction des problèmes } \\
\text { pratiques à résoudre }\end{array}$ & $\begin{array}{l}\text { Jugement de connaissance nécessaire et } \\
\text { relatif en fonction de la problématique } \\
\text { concernée }\end{array}$ \\
\hline Connaissance comme fin en soi & Connaissance utile & $\begin{array}{l}\text { Connaissance comme fin en soi et une } \\
\text { fin utile }\end{array}$ \\
\hline $\begin{array}{l}\text { Dualisme dogmatique } \\
\text { (esprit - corps) }\end{array}$ & $\begin{array}{l}\text { Monisme historique } \\
\text { (vie - pensée) }\end{array}$ & $\begin{array}{l}\text { Dualisme historique } \\
\text { (individuel - collectif) }\end{array}$ \\
\hline
\end{tabular}

Libération par rapport au sommeil dogmatique

Dépassement du rationalisme classique au profit du rationalisme sociologique

Bien que ce cours soit fragmentaire ${ }^{48}$, on peut relever un changement intéressant sur le point discuté jusqu'ici : Durkheim se rapproche de Kant. En effet, c'est sur un mode kantien qu'il classe les règles prises en considération. Il distingue les règles qui valent pour « tous les hommes indistinctement » et se rapportent « à l'homme en général », et 
celles qui «tiennent non à notre qualité générale d'hommes, mais à des qualités particulières que tous les hommes ne présentent pas ${ }^{49}$ ». Parmi ces dernières, il dénombre en particulier les devoirs domestiques, professionnels et citoyens, en un mot, tous les devoirs associés à la constitution de groupes dans une société organisée en Etat. Il est significatif, cependant, qu'il ne distingue pas seulement les devoirs envers l'humanité et les devoirs envers les groupes, mais qu'il subdivise à nouveau les devoirs envers l'humanité en deux groupes. Le premier concerne «les rapport de chacun de nous avec soi-même ». Ces règles, note à présent Durkheim, constituent la "morale individuelle ${ }^{50}$ ». Le second a trait aux « rapports que nous soutenons avec les autres hommes, abstraction faite de tout groupement particulier». En d'autres termes, Durkheim reprend, dans un cadre apparemment universaliste, la distinction entre les devoirs envers soi-même et les devoirs envers les autres. Les devoirs envers soi-même, les règles de la morale individuelle, se voient même attribuer la « fonction de fixer dans la conscience de l'individu les assises fondamentales et générales de toute la morale ». Ils constituent le fondement sur lequel est construit l'édifice des droits et des devoirs. Le couronnement de cet édifice réside dans les devoirs que nous avons envers les autres hommes en tant qu'hommes $^{51}$. Durkheim fonde la construction de sa physique des mœurs et du droit sur ces deux groupes de devoirs envers l'humanité, qui doivent en constituer le cadre. Ces devoirs à l'égard de soi-même et des autres, en effet, sont à ses yeux universels et marquent les deux pôles entre lesquels se déploient les devoirs particuliers, attachés à un groupe. Le primat revient donc ici, à l'évidence, à l'homme indépendant des groupes.

Or, cette argumentation est à bien des égards surprenante, étant donné l'arrière-plan des positions défendues par Durkheim, telles que nous les avons esquissées. D'abord, l'humanité n'est pas un groupe. Le sociologue avait mis l'accent sur ce point dans sa critique de Comte ${ }^{52}$. Mais suivant sa conception, les devoirs qui ne sont pas rattachés à un groupe sont privés de caractère obligatoire. Par suite, de son point de vue, les devoirs envers l'humanité demeurent dévalués par rapport aux devoirs envers le groupe, non seulement dans leurs effets, mais aussi dans leur importance. En second lieu, l'individu ne peut devenir de but en blanc le fondement de la morale. Durkheim ne se lasse jamais de le souligner : le fondement de la morale est le collectif. En troisième lieu, on peut s'étonner du contenu de ces devoirs envers l'humanité. Il convient toutefois ici de faire une restriction: le cours ne traite que des devoirs universels envers les autres, et non des devoirs universels envers soi-même. Les premiers consistent à ne pas tuer l'autre, à respecter sa propriété et à honorer les contrats conclus avec lui ${ }^{53}$. Abstraction faite de l'interdit de tuer, cependant, ce ne sont pas là des devoirs envers l'humanité, mais des devoirs associés à une société particulière, celle que Kant, Hegel et Marx, chacun à sa manière, ont appelé la société civile bourgeoise.

$51 \mathrm{Au}$ demeurant, le cours présente une idée importante, qui va plus loin. On peut, en effet, lire le texte de Durkheim comme une tentative pour institutionnaliser, en même temps que les devoirs particuliers, les devoirs universels, justement parce qu'ils ne sont pas portés par des forces collectives puissantes. En d'autres termes : toutes les sociétés modernes doivent intégrer ces droits de l'humanité dans leur ordre juridique et accorder à ces derniers leurs droits particuliers, qui se trouvent ainsi limités. Ce qui signifie aussi que toutes les sociétés modernes doivent assurer à l'individu des droits de participation et de refus, afin qu'ils puissent choisir de participer à des groupes, mais ne soient pas livrés sans protection à la tyrannie, toujours possible, de ces derniers ${ }^{54}$. Il s'ensuit, enfin, que toutes les sociétés modernes doivent garantir la marge de 
manœuvre de l'individu en autorisant les appartenances à des groupes multiples. Le cours présente un plaidoyer pour un Etat national patriotique de constitution républicaine, pour un Etat laïque, pacifique vis à vis de l'extérieur, pluraliste à l'intérieur, réunissant une multitude de groupes sociaux auto-organisés et doté d'instances intermédiaires entre l'Etat et le citoyen ${ }^{55}$.

Synthèse 5 : Plan de la physique des mœurs et du droit

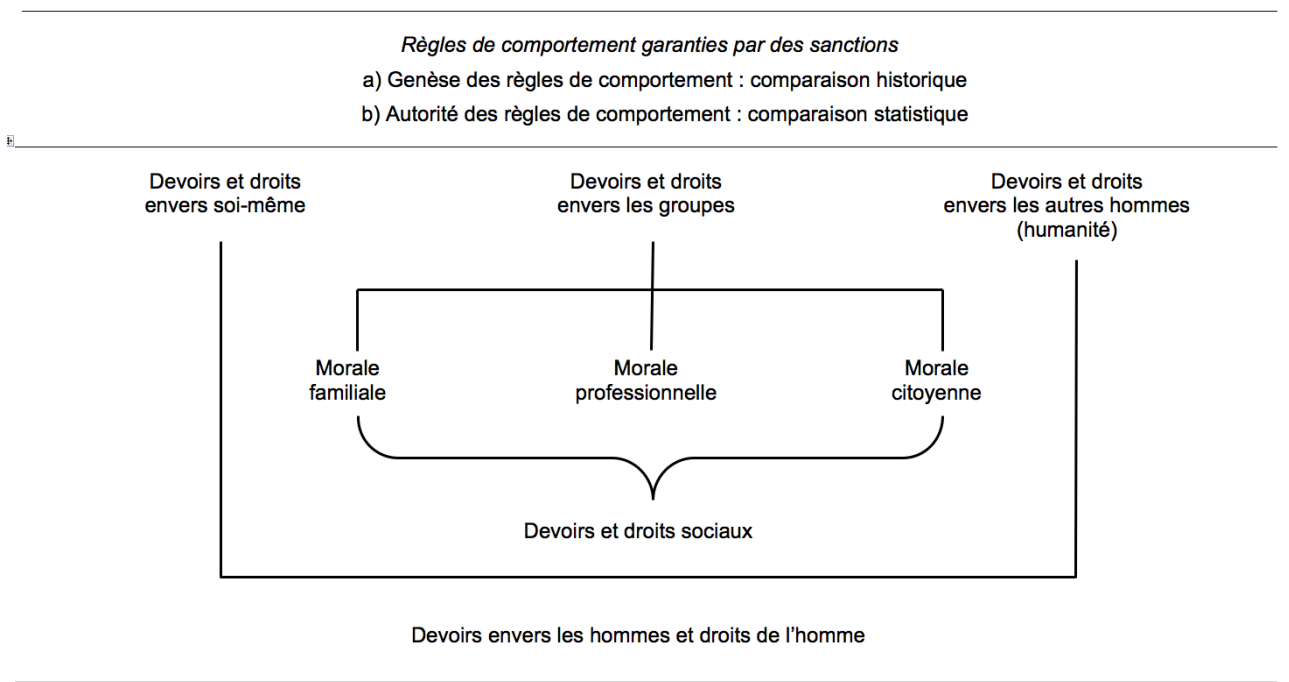

Mais Durkheim ne serait pas Durkheim s'il n'avait pas songé à offrir à l'Etat laïque que la France était, de fait, en train de devenir une consécration religieuse. Le mot d'ordre est ici celui de la religion civique ${ }^{56}$. Si, pour lui, les contrats sont fondés sur des présupposés non contractuels, son propre rationalisme sociologique est fondé, de la même façon, sur des présupposés non rationnels. La morale laïque n'est pas exempte de vestiges religieux, nous l'avons vu, et la pensée scientifique n'est pas entièrement affranchie de la mythologie. On peut en dire autant de l'Etat laïque. Lui aussi construit sa cohésion sur des présupposés non rationnels. Bien entendu, cela ne signifie pas que Durkheim prône un culte de l'Etat - la solution hégélienne, de son point de vue. Il défend un culte de l'individu, la même conception, donc, que Rousseau et Kant, même si elle repose sur un fondement métaphysique (erroné) ${ }^{57}$. Le noyau de cette religion civique est, par suite, l'individualisme moral que Durkheim défendit en intervenant dans l'affaire Dreyfus. Mais le noyau de cette religion civique est aussi la pensée autonome qui conçoit l'individualisme moral comme nécessaire et, par là même, le justifie. L'individualisme moral et la pensée autonome forment le noyau normativocognitif, le fondement sacré de l'Etat laïque. Comme les sociétés tribales totémistes le faisaient déjà, cet Etat doit se célébrer lui-même à intervalles réguliers, afin de donner l'occasion aux citoyens de communier avec ce noyau sacré. Il s'agit de cérémonies au cœur desquelles est placée la "personne humaine». Il importe surtout de rendre présents, par la cérémonie, les devoirs envers l'humanité. Mais cet individualisme moralo-cognitif ne peut recevoir de justification systématique: tout au plus une justification historique. La position de Durkheim aboutit au relativisme. Telle est la conséquence qui résulte de la naturalisation et de la sociologisation de Kant, mais aussi de la tradition du rationalisme métaphysique dans son ensemble. 

en 1914 dans Scientia sous le titre «Le dualisme de la nature humaine et ses conditions sociales ", conçu comme une explication et une défense des Formes élémentaires de la vie religieuse $^{58}$, il fait profession de foi en faveur d'une sociologie de la connaissance et de la morale prenant définitivement congé du rationalisme (métaphysique) de la philosophie occidentale. Selon Durkheim, ce n'est pas un hasard si le rationalisme s'est heurté au dualisme de l'âme et du corps. Cependant, soit il a escamoté le problème, soit il l'a expliqué de façon erronée. La première affirmation correspond à la position du monisme philosophique, la seconde à celle du dualisme philosophique. Une chose est claire : il faut repartir non pas du monisme mais du dualisme, en reprenant seulement la problématique, non la solution qu'il a trouvée. Durkheim distingue ainsi les positions monistes et dualistes, ces dernières se subdivisant quant à elles en positions statiques et dynamiques. Lui-même opte pour un dualisme dynamique qui tient compte des changements historiques dans la relation entre les deux éléments du dualisme, tout en soulignant son caractère constitutif. Quant au monisme, d'après Durkheim il nie en fin de compte le problème : voilà pourquoi il ne saurait être pris en considération par la suite. En effet, il est soit un pur empirisme soit un idéalisme absolu. Pour le pur empirisme, les concepts sont l'élaboration de perceptions sensibles et les actions morales l'élaboration d'intérêts personnels; pour l'idéalisme absolu, les perceptions sensibles sont dérivées de concepts et les actions utiles dérivées des actions morales. Le dualisme, en revanche, reconnaît le problème, mais le traite de manière statique, aussi bien dans sa version ontologique que dans sa version transcendantale. La version ontologique est représentée par le platonisme, qui conçoit un monde des idées préexistant opposé à un monde sans esprit, et un monde du bien préexistant, confronté à un monde mauvais. La version transcendantale est représentée par le kantisme, avec une raison théorique qui s'oppose à la perception sensible, et une raison pratique qui doit vaincre les penchants. Il s'agit là, dans tous les cas, de positions strictement rationalistes. Mais toutes passent à côté du point décisif. Il faut dynamiser le dualisme, qui est supérieur au monisme, mais surtout, il faut en donner une interprétation non pas individualiste mais collectiviste. Pour Durkheim, seule la sociologie de la connaissance et de la morale est en mesure de le faire. En ce sens, elle est située dans la tradition du rationalisme occidental ; cependant, elle ne se contente pas de perpétuer celui-ci : elle le renouvelle.

Par suite, comme Kant, Durkheim voit dans l'homme un homo duplex, mais il ne le considère pas comme un être naturel rationnel, participant à la fois d'un monde intelligible et d'un monde sensible ; il le tient pour un organisme-dans-la-société, placé entre deux pôles de gravitation. Ce qu'il est en tant qu'homme, ce qui le distingue de l'animal, n'est pas produit par son organisme mais par la société. Seule la participation à la vie collective lui permet de dépasser son existence biologique. Il existe bel et bien deux pôles de gravitation, l'organisme et la société, mais ils sont en même temps hiérarchisés. Pour passer des perceptions sensibles basées sur l'organisme aux concepts, des besoins basés sur l'organisme aux besoins moraux, l'homme doit se socialiser et, en même temps, s'individuer. Pour Durkheim, la puissance suprême n'est ni Dieu ni un autre être mystique, mais la société. L'homme est " par nature » asocial et égoïste. Après la naissance physique, il doit connaître une deuxième naissance sociale ${ }^{59}$. De fait, l'organisme humain est constitué de telle façon que cette deuxième naissance est possible. 
Le dualisme dynamique est donc aussi un dualisme hiérarchisé. Ce dernier est constitutif de l'identité humaine. Le platonisme et le kantisme ont bien saisi ce caractère constitutif du dualisme, mais ils l'ont mal interprété. Il trouve son expression la plus aboutie dans l'antagonisme entre sacré et profane. Ce qui est tenu pour sacré change historiquement, comme le montrent les études comparatives, mais l'antagonisme du sacré et du profane ne disparaît jamais.

Nous avons représenté la vision finale de Durkheim dans la figure 6, en prenant en compte les niveaux d'émergence décrits plus haut.

Synthèse 6 : Le dualisme de la nature humaine et ses conditions sociales

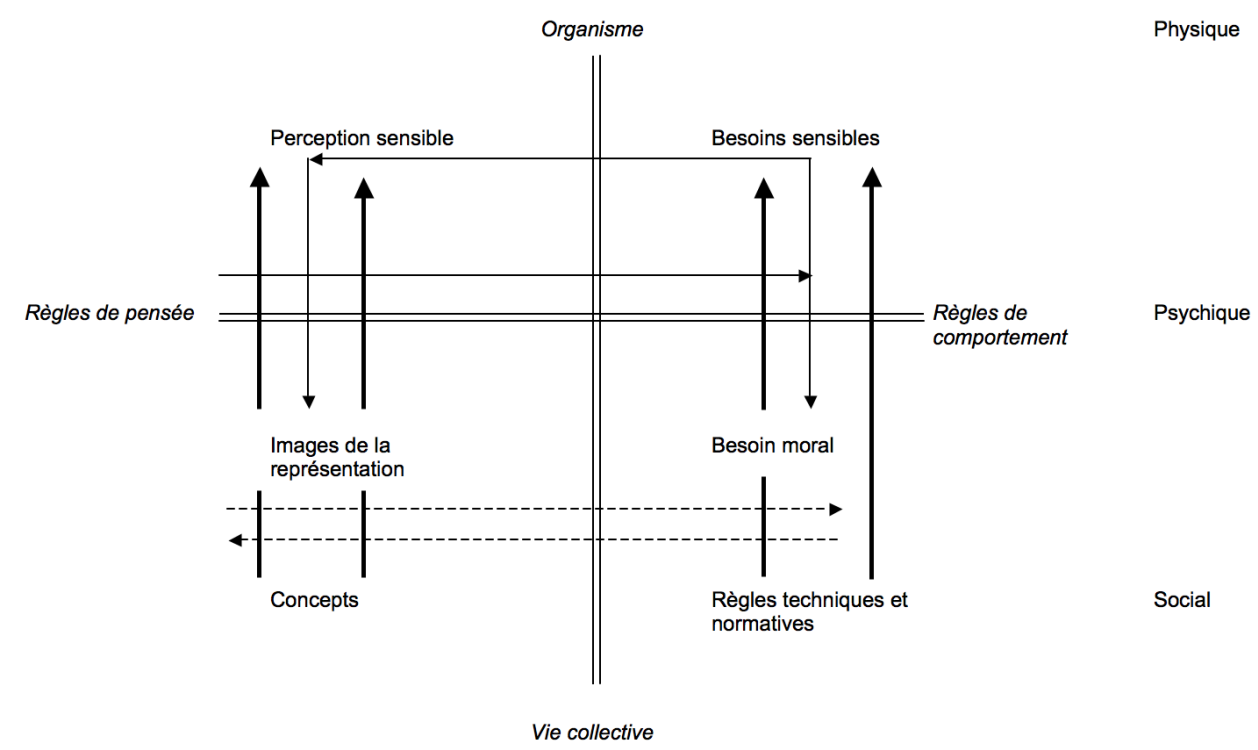

On le voit encore clairement ici: Durkheim traduit le dualisme kantien du monde nouménal et du monde phénoménal en dualisme du monde collectif et du monde individuel. La plupart des idées exposées par Kant dans sa théorie de la connaissance et dans son éthique sont conservées - quoique transformées. Les formes pures de l'intuition et les catégories de Kant deviennent des concepts socialement constitués, mais, tout comme chez Kant, ceux-ci organisent la perception sensible de l'individu. Les impératifs catégoriques de Kant deviennent des règles normatives socialement constituées, mais, tout comme chez Kant, celles-ci déterminent de façon contraignante les besoins sensibles de l'individu. Le sentiment idéal de Kant, le respect de la loi, devient un être-digne-d'aspiration sui generis socialement constitué, mais, tout comme chez Kant, celui-ci ne se constitue que dans l'accomplissement des commandements de la morale. Par suite, on est parfaitement en droit de désigner la position de Durkheim comme un kantisme sociologique. Durkheim ne veut pas compléter Kant, mais le remplacer par sa sociologie de la connaissance et de la morale, et faire ainsi en mieux ce que Kant avait déjà fait.

Cette traduction de Kant, qui se substitue à lui, peut encore être représentée comme suit dans un dernier schéma, à partir de l'exemple de l'acte moral : 
Synthèse 7 : La transformation de Kant par Durkheim

\begin{tabular}{|c|c|c|c|c|c|}
\hline Mobile & $\begin{array}{l}\text { Loi pratique } \\
\text { (impératif } \\
\text { catégorique) }\end{array}$ & $\begin{array}{l}\text { Prescription } \\
\text { pratique } \\
\text { (impératif } \\
\text { hypothétique) }\end{array}$ & Mobile & Règle morale & Règle technique \\
\hline Respect de la loi & Action morale & & $\begin{array}{l}\text { Caractère } \\
\text { souhaitable sui } \\
\text { generis }\end{array}$ & Action morale & \\
\hline \multirow[t]{4}{*}{ Penchant } & & Action utile & $\begin{array}{l}\text { Caractère } \\
\text { souhaitable }\end{array}$ & & Action utile \\
\hline & $\begin{array}{l}\text { Monde } \\
\text { nouménal }\end{array}$ & $\begin{array}{l}\text { Monde } \\
\text { phénoménal }\end{array}$ & & $\begin{array}{l}\text { Vie } \\
\text { collective }\end{array}$ & $\begin{array}{l}\text { Vie } \\
\text { individuelle }\end{array}$ \\
\hline & Autonomie & Hétéronomie & & Altruisme & Egoïsme \\
\hline & \multicolumn{3}{|c|}{$\begin{array}{l}\text { Dualisme de la nature humaine } \\
\text { et ses conditions philosophiques } \\
\text { (l'homme comme être naturel rationnel) }\end{array}$} & \multicolumn{2}{|c|}{$\begin{array}{l}\text { Dualisme de la nature humaine } \\
\text { et ses conditions sociales (l'homme } \\
\text { comme être social) }\end{array}$} \\
\hline
\end{tabular}
que ces cours posaient déjà les bases de cette transformation de la philosophie occidentale et surtout de Kant. Durant sa vie de chercheur, Durkheim poursuivit cette voie de façon conséquente en se consacrant surtout à l'étude de grandes quantités de données. Pour reprendre une distinction de Friedrich Theodor Vischer jugée saisissante par Weber, on pourrait dire que Durkheim fut davantage un promoteur de données qu'un créateur de sens. Il est vrai que l'examen inductif des données, mené pas à pas, ne lui servait finalement qu'à construire une théorie. Durkheim put ainsi inaugurer un programme de recherche qui lui était propre, qui fit école et reste vivant ; sa capacité à bâtir des réseaux n'était pas seule en cause.

us adoptons une perspective systématique et nous plaçons d'un point de vue historique et comparatif, nous pouvons résumer ainsi la position qui fut en fin de compte celle de Durkheim. Sur le plan systématique, il défendit avec constance, par son rationalisme sociologique de la connaissance, l'idée que les notions à l'aide desquelles nous déchiffrons la réalité sont socialement constituées. Durkheim ne nie pas l'existence de stades de la connaissance, depuis la perception sensible jusqu'aux concepts en passant par les représentations, mais pour lui seuls les concepts donnent accès à une vérité impersonnelle, partagée à un niveau intersubjectif, et ils sont exclusivement le produit du collectif. Ils apposent leur marque sur les images de la représentation et sur les perceptions sensibles, alors que l'inverse n'est pas vrai. Avec les concepts socialement constitués, nous ne nous contentons pas, au demeurant, de reproduire la réalité, nous produisons des développements nouveaux. C'est la raison pour laquelle la connaissance conceptuelle n'est jamais seulement utile mais est toujours aussi une fin en soi, spéculative. Bien qu'elle demeure toujours attachée à un contexte social, elle contient des anticipations. Nous ne produisons donc pas seulement des modèles de la réalité, mais aussi des modèles pour la réalité.

D'un point de vue systématique, avec son rationalisme sociologique des mours et du droit, Durkheim insiste sur le fait que les règles de comportement qui régissent nos actions sont socialement constituées. Elles sont en existe de deux sortes, les règles techniques et les règles normatives, et ces dernières peuvent se subdiviser à leur tour en règles religieuses, morales et juridiques. Les règles normatives priment sur les règles techniques. Les premières participent dans une certaine mesure du sacré, tandis que les secondes en sont exclues. Par conséquent, nos actions sont divisées en deux types, les actions normatives-altruistes et les actions techniques-égoïstes. L'action consiste en 
premier lieu à se conformer à des règles, mais nous donnons également à celles-ci de nouveaux développements. Il s'agit là moins d'une affaire individuelle que d'une affaire collective. C'est surtout dans les cérémonies, lorsque les âmes individuelles peuvent fusionner avec l'âme du groupe, que de nouveaux idéaux peuvent voir le jour. Ils doivent ensuite être institutionnalisés et internalisés ${ }^{60}$. Le plus souvent, ces idéaux sont incompatibles avec le statu quo. Le siège de la créativité de l'action est donc le groupe. La "créativité » de l'individu apparaît davantage comme un écart. Là encore, nous avons affaire à un dualisme hiérarchisé : ce n'est pas la conscience individuelle qui pénètre la conscience collective, mais l'inverse.

D'un point de vue historique et comparatif, le rationalisme sociologique de la connaissance est lui-même le produit d'une évolution. D'une pensée mythologique et dogmatique, on est passé à une pensée scientifique et critique. La pensée ne s'est pas détachée de ses origines sociales, mais elle s'est affranchie de son contenu social. Dans la mise en ordre du monde par la pensée, dans les classifications, les transmutations ont fait place aux distinctions logiques, qui permettent, par exemple, de ne pas confondre causalité et sanction ou niveau individuel et niveau collectif. De surcroît, la pensée s'affranchit ainsi de la pression immédiate de l'action.

D'un point de vue historique et comparatif, le rationalisme sociologique des mours et $d u$ droit est également le produit d'une évolution. D'une morale religieuse hétéronome, on est passé à une morale laïque autonome. Il en a résulté, pour l'individu, un élargissement de sa marge de liberté et un affranchissement par rapport à la pression immédiate du groupe. Un individualisme moral est ainsi apparu, associé à des droits et des devoirs universels, mais aussi à un culte de l'individu. C'est aussi là une position normative que Durkheim reprend à son compte. Aujourd'hui, [...] nous parlerions d'une défense communautariste du libéralisme.

Au demeurant, il ressort également de ce résumé que Durkheim ne parvient pas à se détacher du modèle dichotomique de ses prédécesseurs et contemporains. Il le modifie considérablement, mais toute son œuvre demeure marquée par une typologie bipolaire interprétée en termes évolutionnistes. De surcroît, la conception qui organise la nature en niveaux d'émergence, associée à la thèse du dualisme constitutif de la nature humaine, ouvre certes la voie à une analyse à plusieurs niveaux, mais l'interaction entre ces derniers est interprétée de différentes manières et, en fin de compte, c'est le modèle hiérarchique qui prévaut. La conscience individuelle est pénétrée par la contrainte de la conscience collective. Une "downward causation" s'exerce et fait courir, par suite, le danger d'une "downward conflation ${ }^{61}$. Bien qu'il existe des ouvertures vers une reconnaissance des droits spécifiques de l'action individuelle, Durkheim ne cesse de retomber, d'un point de vue méthodologique, dans le holisme massif des Règles de la méthode sociologique. Le fondement microsociologique des processus macrosociologiques demeure obscur. De surcroit, la relation entre l'action technique égoïste et l'action normative altruiste pose problème. En effet, ces types d'action sont ancrés dans deux niveaux différents, parce qu'ils sont associés à la distinction entre sacré et profane. Toute action fondée sur une stimulation, par exemple l'action économique, est associée au domaine profane. Par là, cependant, elle est en un sens paradoxalement "désociologisée». En fin de compte, on peut se demander quel avantage on peut trouver à remplacer la théorie de la connaissance par une sociologie de la connaissance et la philosophie morale par une sociologie de la morale. Car cela conduit, en effet, comme toutes les tentatives de ce type, à un 
relativisme fondamental, qui demeure toujours contradictoire. Pour l'objet qui nous occupe, toutefois, le point décisif est le suivant : Durkheim ne parvient pas à construire une théorie de l'action convaincante. Sur ce point, le jeune Marx était allé plus loin, avec son modèle de l'activité. La thèse durkheimienne de la correspondance entre structure et culture ne dépasse pas non plus significativement le modèle de la base et de la superstructure proposé par Marx ${ }^{62}$. [...]

\section{BIBLIOGRAPHIE}

Archer, M. S. (1988) : Culture and Agency. The Place of Culture in Social Theory, Cambridge :

Cambridge University Press.

Bellah, R. N. (1970) : Beyond Belief. Essays on Religion in the Post-Traditional World, New York : Harper \& Row.

Cladis, M. S. (1992) : A Communautarian Defense of Liberalism. Emile Durkheim and Contemporary Social Theory, Stanford : Stanford University Press.

Clark, T. N. (1981) : « Die Durkheim-Schule und die Universität », in : Lepenies (1981), p. 157-187.

Durkheim, E. (1925) : L'Education morale. Mémoires de l'Année sociologique, Paris : Alcan.

Durkheim, E. (1926) : « Pédagogie et sociologie », in : Id. : Education et sociologie, $2^{\mathrm{e}}$ éd., Paris : Alcan, p. 104-133.

Durkheim, E. (1938) : L'Evolution pédagogique en France, introd. par M. Halbwachs, Paris : Alcan.

Durkheim, E. (1955) : Pragmatisme et sociologie. Cours professé à la Sorbonne pendant l'année universitaire 1913-14. Texte restitué d'après les notes d'étudiants par Armand Cuvilier, Paris : Vrin.

Durkheim, E. (1969) : Journal sociologique par Emile Durkheim, Paris : PUF.

Durkheim, E. (1970 [1914]) : « Le dualisme de la nature humaine », dans : La science sociale et l'action, éd. par J. C. Filloux, Paris : PUF, p. 314-332 ; ed. or. : (1914) in : Scientia, XV, p. 206-221.

Durkheim, E. (1987) : Schriften zur Soziologie der Erkenntnis, éd. par H. Joas, Francfort-sur-le-Main : Suhrkamp.

Durkheim, E. (1990 [1912]) : Les Formes élémentaires de la vie religieuse. Le système totémique en Australie, Paris : PUF.

Durkheim, E. (1990) : Leçons de sociologie, Paris : PUF.

Durkheim, E. (1994) : La Division du travail social, $3^{\mathrm{e}}$ éd., Paris : PUF.

Joas, H. (1985) : « Durkheim und der Pragmatismus. Bewußtseinspsychologie und die soziale Konstitution von Kategorien », Kölner Zeitschrift für Soziologie und Sozialpsychologie, 37 (3), 1985, p. 411-430 ; repris dans : Durkheim (1987), p. 257-288.

Kant, E. (1869) : Critique de la raison pure, trad. J. Barni, Paris : Germer-Baillière.

Kant, E. (1960) : Fondements de la métaphysique des mœurs, trad. V. Delbos, Paris : Delagrave. 
Kohlberg, L. (1997) : Die Psychologie der Moralentwicklung, trad. de l'anglais, Francfort-sur-le-Main : Suhrkamp ; [la réf. citée en note 39 correspond à l'article « Moral stages and moralization: the cognitif-developmental approach » (1976), NDLR].

Kohlberg, L. (2000) : Psychologie der Lebensspanne, éd. et intr. par W. Althof et D. Garz, trad. D. Garz, Francfort-sur-le-Main: Suhrkamp ; introduction : p. 18-66.

Lepenies, W. (éd.) (1981) : Geschichte der Soziologie. Studien zur kognitiven, sozialen und historischen Identität einer Disziplin, t. 2, Francfort-sur-le-Main : Suhrkamp.

Lepenies, W. (1990) : Les Trois cultures : entre science et littérature, l'avènement de la sociologie, trad. de l'allemand par H. Plard, Paris : Editions de la Maison des sciences de l'homme.

Lévi-Strauss, C. (1962) : Le totémisme aujourd'hui, Paris : PUF.

Piaget, J. (1932) : Le Jugement moral chez l'enfant, Paris : Alcan.

Schluchter, W. (2000) : « Über Individualismus », in : Id. : Individualismus, Verantwortungsethik und Vielfalt, Weilerswist : Velbrück Wissenschaft.

Wright, G. (1987) : France in Modern Times. From the Enlightenment to the Present, $4^{\mathrm{e}}$ éd., vol. 3 : The Rooting of the Republican System 1870-1919, New York / Londres : W. W. Norton Company.

\section{NOTES}

1. L'Année sociologique, dont le volume I fut publié par Durkheim en $1896 / 97$, publia dans son volume II, en 1897/98, au titre de "mémoire original ", son article "De la définition des phénomènes religieux». Dans la «Préface» à ce volume, il faisait observer que c'était la deuxième fois qu'une étude de sociologie des religions était placée en tête de l'Année sociologique. Dans le volume I, Durkheim s'était ainsi intéressé au tabou de l'inceste («La prohibition de l'inceste et ses origines »). Le lecteur, remarquait-il, s'étonnait peut-être qu'une telle place fût réservée au traitement des phénomènes religieux, mais «c'est qu'ils sont le germe d'où tous les autres - ou, tout au moins, presque tous les autres - sont dérivés. La religion contient en elle, dès le principe, mais à l'état confus, tous les éléments qui, en se dissociant, en se déterminant, en se combinant de mille manières avec eux-mêmes, ont donné naissance aux diverses manifestations de la vie collective. » (Durkheim [1969], p. 138).

2. Durkheim (1990 [1912]).

3. Durkheim (1990 [1912]), p. 22.

4. Durkheim (1990 [1912]), p. 12.

5. Durkheim (1990 [1912]), p. 50.

6. Durkheim (1990 [1912]), p. 51.

7. Au demeurant, il faudrait établir une distinction entre institution et organisation. Durkheim confond les deux.

8. Durkheim (1990 [1912]), p. 65 (en italique dans l'original).

9. Ibid.

10. On peut ainsi lire dans Durkheim (1990 [1912]), p. 61 : « Il n'existe pas d'Église magique.Entre le magicien et les individus qui le consultent, comme entre ces individus eux-mêmes, il n'y a pas de liens durables qui en fassent les membres d'un même corps moral, comparable à celui que forment les fidèles d'un même dieu, les observateurs d'un même culte. »

11. Weber n'aurait pas dit lui non plus qu'une religion exigeait nécessairement une Eglise. Comme on le sait, il établissait une distinction entre Eglise et secte et reconnaissait en outre l'existence d'autres types de communautés religieuses.

12. Durkheim (1990 [1912]), p. 123 sq. 
13. L'institution de L'Année sociologique a aussi pour objectif, aux yeux de Durkheim, de faire perdre peu à peu aux sociologues la confiance qu'ils plaçaient dans la méthode dialectique : « En effet, les connaissances qu'un sociologue doit posséder, s'il ne veut pas se livrer à un vain exercice de dialectique, sont tellement étendues et variées, les faits sont si nombreux, épars de tant de côtés qu'on a grand mal à les trouver et qu'on risque d'en omettre d'essentiels » (Durkheim [1969],p. 31).

14. Cf. Lévi-Strauss (1962).

15. Durkheim (1990 [1912]), p. 5.

16. On a proposé de diviser en quatre phases la période allant de 1870 à 1919 , qui fut la période de référence de Durkheim dans sa perception de la société française : la république monarchique (1870-1879), la république opportuniste (1879-1898), la république radicale (1898-1914) et la république "en guerre » (1914-1919). Né en 1858 et mort en 1917, Durkheim vécut ces quatre phases, même si la période de transition entre la république opportuniste et la république radicale fut particulièrement importante pour lui. Sur cette chronologie, voir Wright (1987), p. 209 sq.

17. Dans Durkheim (1925), p. 116, on peut lire: «Il n'y a pas dans l'histoire de crise aussi grave que celle où les sociétés européennes sont engagées depuis plus d'un siècle. » On pourrait citer d'autres passages.

18. Sur ce point, voir Cladis (1992) et Schluchter (2000), p. 9 sq.

19. Voir Durkheim (1969), p. 34, pour la préface au volume I de L'Année sociologique. Plus tard, à partir du volume XI, les « Mémoires originaux » furent publiés à part, dans une "Collection des travaux de l'Année sociologique ». La part réservée aux « Notes critiques » fut ainsi augmentée et le délai entre la parution du livre et celle du compte rendu étendu à trois ans. Voir la Préface au volume XI in : Durkheim (1969), p. 625.

20. L'année commençait en juillet, et c'était la date de publication qui était prise en compte. C'est la raison pour laquelle les premiers volumes s'étendent sur deux ans (1896/97, 1897/98, 1898/99, etc.).

21. Elles ont été rassemblées dans le Journal sociologique.

22. La stratégie durkheimienne de fondation d'une école a été étudiée en détail par les historiens des sciences. On trouvera d'importantes contributions sur le sujet dans Lepenies (1981), notamment les articles de Terry N. Clark, Victor Karady et Philippe Besnard. Clark note au sujet de l'équipe de collaborateurs de L'Année sociologique: «Durkheim et la plupart des autres collaborateurs de L'Année sociologique étaient prédestinés, par leurs origines, à une carrière universitaire. Le candidat idéaltypique avait obtenu une excellente mention au baccalauréat puis fait des études à l'Ecole Normale Supérieure ; il avait été reçu à l'agrégation de philosophie ; il avait enseigné cette discipline dans un lycée de province, puis obtenu une bourse pour séjourner en Allemagne; il avait ensuite obtenu le doctorat-ès-lettres. Les caractéristiques personnelles importante, en dehors des critères académiques, étaient une origine familiale petite-bourgeoise (idéalement, un père instituteur), un dévouement passionné à la République, un anti-cléricalisme militant et, en politique, une préférence pour les radicaux-socialistes ou les socialistes. L'origine juive de Durkheim et de certains de ses collègues ne semble pas avoir joué un rôle déterminant au sein de l'université ; la question religieuse prédominante était la lutte entre cléricalisme et anti-cléricalisme » (Terry N. Clark, « Die Durkheim-Schule und die Universität», p. 161). Cette caractérisation, au demeurant, est trop calquée sur le parcours personnel de Durkheim.

23. Durkheim (1925). Durkheim remplaçait le pédagogue Ferdinand Buisson, dont il reprit définitivement la chaire en 1906. Dans ce contexte, nous renvoyons aussi au cours de 1904/1905 «Histoire de l'enseignement en France», paru à titre posthume sous le titre L'Evolution pédagogique en France, avec une introduction de Maurice Halbwachs (Durkheim [1938]). Cette étude d'histoire de la morale et des institutions a pour but de retracer la genèse du culte de la personne humaine. A ce titre, elle approfondit la thèse historique présentée dans le cours sur 
l'éducation morale. On peut ainsi lire, p. 372 : «Toute notre morale actuelle est dominée par le culte de la personne humaine ; les Romains et les Grecs l'ignoraient. »

24. Sur ce point, voir Lepenies (1990), p. 45 sq. Selon Lepenies, la Nouvelle Sorbonne était attaquée de trois côtés à la fois : par la droite, alliée à l'Action Française, qui se réclamait en partie du dernier Auguste Comte; par la philosophie de la vie d'Henri Bergson; et par Charles Péguy, qui défendait une sorte de socialisme mythologisant et qui, à la différence de Durkheim, regardait la sociologie et la démocratie comme antagonistes. Les attaques visaient surtout Durkheim et l'école durkheimienne, auxquels on reprochait en outre d'être à la solde de l'Allemagne. La campagne de diffamation atteignit son point culminant en 1910.

25. Durkheim (1925), p. 3 sq.

26. Durkheim (1925), p. 114 sq.

27. Il ne s'agit donc pas d'un retour à Auguste Comte.

28. Durkheim (1925), p. 130.

29. Piaget (1932), p. 2.

30. Cette classification tient au fait que le développement moral va de pair avec le développement cognitif et que tous deux dépendent de l'âge. Cf. Kohlberg (1997); (2000), p. 18 sq., en particulier p. 27 sq.

31. Kant (1960), p. 103.

32. Kant (1960), p. 156.

33. Kant, Critique de la raison pratique, § 8 (trad. Barni), cité par Durkheim (1925), p. 124.

34. Durkheim (1925), p. 125.

35. Durkheim (1925), p. 126.

36. Durkheim (1925), p. 47.

37. Durkheim (1925), p. 135.

38. Piaget, Le Jugement moral chez l'enfant, chap. IV.

39. Cf. Kohlberg (1997), p. 130. Le descriptif proposé par Kohlberg dans ce passage a été ici présenté en abrégé.

40. Durkheim (1925), p. 134.

41. Durkheim (1925), p. 126.

42. Durkheim (1955).

43. C'est là une autre allusion à Kant. On le sait, celui-ci fut réveillé de son sommeil dogmatique par les écrits de David Hume. Ce n'est qu'à compter de ce moment qu'il fut en mesure de formuler sa critique de la métaphysique traditionnelle, une critique de la raison pure à l'aide de la raison.

44. Durkheim (1955), p.175 sq.

45. Sur ce point, voir Joas (1985). En Europe, le pragmatisme a longtemps fait l'objet d'une présentation caricaturale. C'est le mérite de Hans Joas que de l'avoir remise en cause. Il repère chez Durkheim beaucoup de déformations, et se demande dans quelle mesure elles sont intentionnelles ou dues à une méconnaissance de ce courant. Sur ce point, voir Joas (1985), p. 414. 46. Durkheim (1955), p. 169 sq.

47. Durkheim distingue là encore les sanctions répressives, restitutives et appellatives, ou les sanctions du droit pénal, du droit civil et de la morale. Cf. Durkheim (1990), p. 42.

48. Manquent les passages sur les devoirs envers soi-même et sur les devoirs envers la famille.

49. Durkheim (1990), p. 43.

50. Ibid.

51. Cf. Durkheim (1990), p. 43 : «Les règles de la morale individuelle ont en effet pour fonction de fixer dans la conscience de l'individu les assises fondamentales et générales de toute la morale ; c'est sur ces assises que tout le reste repose. Au contraire, les règles qui déterminent les devoirs que les hommes ont les uns envers les autres par cela seul qu'ils sont hommes, sont la partie culminante de l'éthique. » 
52. Durkheim songe à la notion de civilisation comme maillon intermédiaire entre l'humanité et l'Etat national. Cf. la note rédigée avec Marcel Mauss : « Note sur la notion de civilisation » dans Durkheim (1969), p. 681 sq. Elle implique en même temps une modification de la critique de Comte.

53. Durkheim (1990), p. 152 sq.

54. Sur ce point, voir notamment les remarques de la cinquième leçon, Durkheim (1990), p. 91 sq. En accord avec sa théorie, Durkheim constate que ces droits sont naturellement conférés par l'Etat, donc par le collectif, mais ne sont pas pré-étatiques, comme l'admet la tradition du droit naturel.

55. Dans ce domaine, Durkheim attache une importance particulière aux groupes professionnels. Déjà, dans la préface à la deuxième édition de La Division du travail, il évoquait le rôle de ces derniers pour la cohésion de la société de la division du travail. Il revient sur cette idée dans son cours. Au demeurant, il est très soucieux de distinguer les groupes professionnels modernes des groupes pré-modernes, corporations et guildes. Cependant, son modèle de constitution politique moderne est moins orienté vers un Etat de partis que vers un Etat de corporations.

56. L'important débat relatif à cette question va de Rousseau à Robert Bellah en passant par Durkheim. Cf. Bellah (1970), chap. 9.

57. Durkheim (1990), quatrième leçon, p. 79 sq., en particulier p. 88 sq.

58. L'original est reproduit in Durkheim (1970), p. 314-332.

59. Voir la leçon inaugurale de Durkheim à la Sorbonne, succédant à Ferdinand Buisson en 1902 (Durkheim [1926]), en particulier p. 119 sq.

60. Cf. Durkheim (1970), p. 329 : «Mais ces idéaux, produit de la vie en groupe, ne peuvent se constituer, ni surtout subsister, sans pénétrer dans les consciences individuelles et sans s'y organiser d'une manière durable. Ces grandes conceptions religieuses, morales, intellectuelles que les sociétés tirent de leur sein pendant leurs périodes d'effervescence créatrice, les individus les emportent en eux une fois que le groupe s'est dissous, que la communion sociale a fait son œuvre.»

61. Sur ces concepts, voir Archer (1988), p. XII sq. et 25 sq.

62. Durkheim s'intéressa beaucoup au socialisme comme courant théorique et comme mouvement pratique. Notamment dans un cours donné de novembre 1895 à mai 1896 à Bordeaux. Il en publia certains extraits en revue. Ceux-ci furent plus tard rassemblés et complétés. Le résultat fut le livre Le Socialisme, édité par Marcel Mauss en 1928, assorti d'une introduction. Dans un premier temps, Durkheim avait l'intention d'écrire une étude sur les relations entre individualisme et socialisme, et c'est ce qui devint La Division du travail. Mais son intérêt pour le socialisme ne faiblit pas, notamment en raison de l'importance de Saint-Simon pour Comte et pour lui-même. Il souhaitait écrire une histoire du socialisme qui aurait débuté avec Saint-Simon, et dans laquelle il aurait également été question de Marx, du socialisme allemand et de Proudhon. Il en resta cependant à la critique de Saint-Simon, de ses prédécesseurs et de son école. Une confrontation théorique approfondie avec Marx fait défaut.

\section{INDEX}

Schlüsselwörter : Politik, Religion, Max Weber, Religionssoziologie

Mots-clés : politique, religion, Max Weber, sociologie religieuse 


\section{AUTEURS}

\section{WOLFGANG SCHLUCHTER}

Wolfgang Schluchter est professeur à l'Université d'Heidelberg. Pour plus d'informations, voir la notice suivante. 